\title{
Non-Invasive Assessment of Dairy Products Using Spatially Resolved Diffuse Reflectance Spectroscopy
}

Abildgaard, Otto Højager Attermann; Kamran, Faisal; Dahl, Anders Bjorholm; Skytte, Jacob Lercke; Nielsen, Frederik D. ; Thomsen, Carsten Lilholt; Andersen, Peter E.; Larsen, Rasmus; Frisvad, Jeppe Revall

Published in:

Applied Spectroscopy

Link to article, DOI:

10.1366/14-07529

Publication date:

2015

Document Version

Peer reviewed version

Link back to DTU Orbit

Citation $(A P A)$ :

Abildgaard, O. H. A., Kamran, F., Dahl, A. B., Skytte, J. L., Nielsen, F. D., Thomsen, C. L., Andersen, P. E., Larsen, R., \& Frisvad, J. R. (2015). Non-Invasive Assessment of Dairy Products Using Spatially Resolved

Diffuse Reflectance Spectroscopy. Applied Spectroscopy, 69(9), 1096-1105. https://doi.org/10.1366/14-07529

\section{General rights}

Copyright and moral rights for the publications made accessible in the public portal are retained by the authors and/or other copyright owners and it is a condition of accessing publications that users recognise and abide by the legal requirements associated with these rights.

- Users may download and print one copy of any publication from the public portal for the purpose of private study or research.

- You may not further distribute the material or use it for any profit-making activity or commercial gain

- You may freely distribute the URL identifying the publication in the public portal 


\title{
Non-Invasive Assessment of Dairy Products Using Spatially Resolved Diffuse Reflectance Spectroscopy
}

\author{
Otto H. A. Abildgaard, ${ }^{1}$ Faisal Kamran, ${ }^{2}$ Anders B. Dahl, ${ }^{1}$ Jacob L. Skytte, ${ }^{1}$ Frederik D. \\ Nielsen, ${ }^{3}$ Carsten L. Thomsen, ${ }^{3}$ Peter E. Andersen, ${ }^{2}$ Rasmus Larsen, ${ }^{1}$ and Jeppe R. Frisvad ${ }^{1, *}$ \\ ${ }^{1}$ Department of Applied Mathematics and Computer Science, Technical University of Denmark, 2800 Kgs. Lyngby, Denmark \\ ${ }^{2}$ Department of Photonics Engineering, Technical University of Denmark, 4000 Roskilde, Denmark \\ ${ }^{3}$ NKT Photonics A/S, Blokken 84, 3460 Birkerфd, Denmark
}

\begin{abstract}
The quality of a dairy product is largely determined by its microstructure which also affects its optical properties. Consequently, an assessment of the optical properties during production may be part of a feedback system for ensuring the quality of the production process. This paper presents a novel camerabased measurement technique that enables robust quantification of a wide range of reduced scattering coefficients and absorption coefficients. Measurements are based on hyperspectral images of diffuse reflectance in the wavelength range of 470 to $1020 \mathbf{n m}$. The optical properties of commercially available milk and yogurt products with three different levels of fat content are measured. These constitute a relevant range of products at a dairy plant. The measured reduced scattering properties of the samples are presented, and show a clear discrimination between levels of fat contents as well as fermentation. The presented measurement technique and method of analysis is thus suitable for a rapid, noncontact, and non-invasive inspection that can deduce physically interpretable properties.
\end{abstract}

Index Headings: Non-invasive spectroscopy; Oblique incidence reflectometry; Diffuse reflectance; Absorption; Reduced scattering; Turbid media; Milk; Yogurt.

\section{INTRODUCTION}

Monitoring microstructures in food and beverages is key to enhancing manufacturing process flexibility and efficiency while also improving the quality and safety of the products. ${ }^{1,2}$ For yogurt products, the microstructure, as defined by Bijnen et al., ${ }^{1}$ is a central component in consumers' perception of mouthfeel and thus their acceptance of the product. ${ }^{3}$ The microstructure is formed from an aggregation of protein during the fermentation process. ${ }^{4}$ It is known that reduced scattering properties, ${ }^{5}$ scattering phase function ${ }^{6}$ and the speckle characteristics $^{7-9}$ are affected by the microstructure. Inspection tools which quantify any of these effects may therefore reduce manufacturing costs and improve product quality. ${ }^{10,11}$

The reduced scattering properties can be calculated from steady-state diffuse reflectance measurements as demonstrated by Farrell et al. ${ }^{12}$ This enables measurement of the re-

\footnotetext{
* Corresponding author: jerf@dtu.dk

(C2015 Society for Applied Spectroscopy. One print or electronic copy may be made for personal use only. Systematic reproduction and distribution, duplication of any material in this paper for a fee or for commercial purposes, or modifications of the content of this paper are prohibited.
}

duced scattering and absorption properties. However, this requires measurement of absolute intensities which is infeasible for in-line measurements. ${ }^{13}$ To solve this problem, the technique has been modified by Wang and Jacques ${ }^{14}$ into oblique incidence reflectometry (OIR). The requirement of absolute intensities is avoided by instead resolving both single and multiple scattering of the light. The drawback of using OIR is that the range of optical properties which can be inspected, is limited directly by the finite spatial resolution and the field of view of the detector system. This limits the ability to resolve optically dense or dilute media.

Oblique incidence reflectometry has mainly been used for inspection of tissues, which span a relatively small range of optical properties. ${ }^{15-18}$ Tissue measurements are obtained using a fiber probe. Such probes are convenient for measuring the skin properties of live subjects, but also limited to the narrow range of optical properties of skin. To make the measurement of a milk fermentation process more feasible, a wider range of optical properties must be covered. ${ }^{5}$ This is most feasible with camera-based measurements which has a much higher spatial resolution, typically a few thousand detectors along the diagonal of a modern charged coupled device (CCD) in comparison to 12 for fiber probes. ${ }^{18}$ The original OIR technique ${ }^{14}$ was camera-based and demonstrated the potential of the technique for measuring the reduced scattering $\mu_{s}^{\prime}$. Measurement of the absorption coefficient $\mu_{a}$ and the spectral dependencies was not introduced until after the shift to systems based on fiber probes. ${ }^{15,19}$ Some work has been done in camera-based OIR, ${ }^{20,21}$ where the challenges summarised in Table I were identified. In this paper, the markers C. 1 to C. 7 refer to these challenges.

The objective of this paper is to demonstrate an inspection tool for quantifying the reduced scattering properties as well as the absorption properties of dairy products suitable for inline process inspection. To ensure industrial relevance, the presented inspection tool is applied to commercially available milk and yogurt products with three different levels of fat content. In comparison to previous camera-based instruments for OIR, the presented instrument provides spectrally resolved measurements. It is described how the challenges C. 1 to C.7 occur in the presented measurement method and how they are solved. The challenges are dealt with in a sample independent manner, so that the solutions are directly transferable to other applications. Finally, the measured reduced scattering properties show a clear distinction between fermented and non-fermented milk which is promising for future in-line inspection of the milk fermentation process. 
TABLE I. Challenges in the use of camera-based OIR measurement reported in the literature.

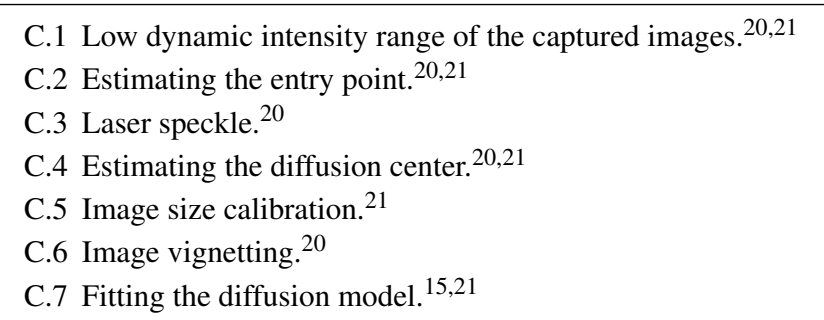

Related Work. The measurement principle of OIR is illustrated in Fig. 1. In Fig. 1a, the light beam is obliquely incident on the sample surface. Inside the sample the light is scattered in different directions, and some emerges from the surface as diffuse reflectance. The highest intensity is closest to the entry point, and the intensity declines further away. Most of the reflectance can be described as two components, and this is illustrated in Fig. 1b. Here, a profile of the reflectance values is illustrated; the line goes through the entry point in the direction of the incident beam. The first component (red) is most intense. This is a region where light has only experienced a few scattering events. The second component (blue) is the low-intensity contribution far from the entry point. This is the region where the reflectance may be described by a diffusion process. Because light enters the sample at an oblique angle, the reflectance profile is elongated, and more light is emitted from the forward direction. This is seen from the so-called center line between the two sides of the reflectance profile, marked by a black line in Fig. 1b. The center line is the geometrical center of the reflectance profile for different intensity levels. At high intensities, the line is located at the entry point, and at low intensities the line is shifted forward towards a new location. The displacement of the two symmetry points for the reflectance profile is denoted $\Delta \mathrm{x}$.

In OIR, the relation between $\Delta \mathrm{x}$ and the light transport diffusion coefficient $D$ is modelled as follows: ${ }^{15}$

$$
D=\frac{\Delta \mathrm{x}}{3 \sin \theta_{t}}=\frac{n \Delta \mathrm{x}}{3 \sin \theta_{i}},
$$

where $\theta_{t}$ is the angle of refraction, and this depends on the angle of incidence $\theta_{i}$ and the relative index of refraction $n$ as specified by the law of refraction (Snell's law). The relative index of refraction is $n=n_{\text {sample }} / n_{\text {ambient }}$. In the present work, the surrounding medium is air $\left(n_{\text {ambient }}=1\right)$ and the refractive index of milk is assumed to be $1.347 .{ }^{22} \mathrm{D}$ may also be related to the transport mean free path (mfp') as $3 D \approx 1 \mathrm{mfp}$ ', which gives the simple interpretation that light on average travels this distance into the sample before being scattered. ${ }^{15}$

It has been found, by comparison with Monte Carlo models ${ }^{15}$ that the diffusion coefficient can be expressed as

$$
D \approx \frac{1}{3\left(\mu_{\mathrm{s}}^{\prime}+0.35 \mu_{\mathrm{a}}\right)} .
$$

Here $\mu_{\mathrm{s}}^{\prime}$ and $\mu_{\mathrm{a}}$ are the reduced scattering coefficient and the absorption coefficient, respectively, and this provides the first parametrisation of the optical properties. Equation 2 can be considered a simplification of the expression for $D$ reported by Ripoll et al. ${ }^{23}$ (a)

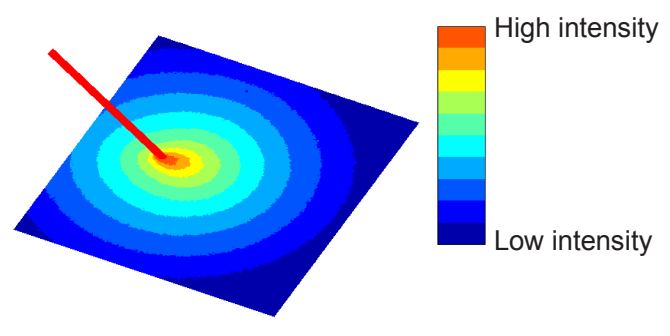

(b)

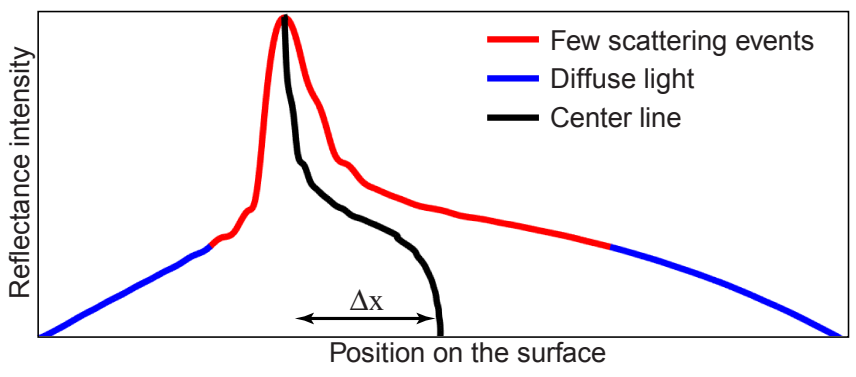

Fig. 1. The measurement principle in OIR. Conceptual illustration of (a) reflectance intensity of a sample surface illuminated by a laser beam (red line) and (b) reflectance of the sample along the entry direction and through the entry point. The profile is asymmetric and shifts by a distance $\Delta \mathrm{x}$ from top to bottom.

The light becomes diffuse as one moves away from the point of entry. The light is therefore modelled using a displaced diffusion dipole as illustrated in Fig. 2. The expression by Farrell et al. ${ }^{12}$ is used but with the dipole (the positive and negative point sources) shifted horizontally by the distance $\Delta \mathrm{x}$ and shifted toward the sample surface according to the modified expression for $D$. The diffuse reflectance at a point $\mathbf{r}=(x, y, z=0)$ on the surface then becomes ${ }^{15}$

$$
\begin{aligned}
\mathrm{R}(\mathbf{r})= & 3 D\left(\mu_{\mathrm{eff}} \rho_{1}+1\right) \frac{e^{-\rho_{1} \mu_{\mathrm{eff}}}}{4 \pi \rho_{1}^{3}} \\
& +(3 D+4 A D)\left(\mu_{\mathrm{eff}} \rho_{2}+1\right) \frac{e^{-\rho_{2} \mu_{\mathrm{eff}}}}{4 \pi \rho_{2}^{3}},
\end{aligned}
$$

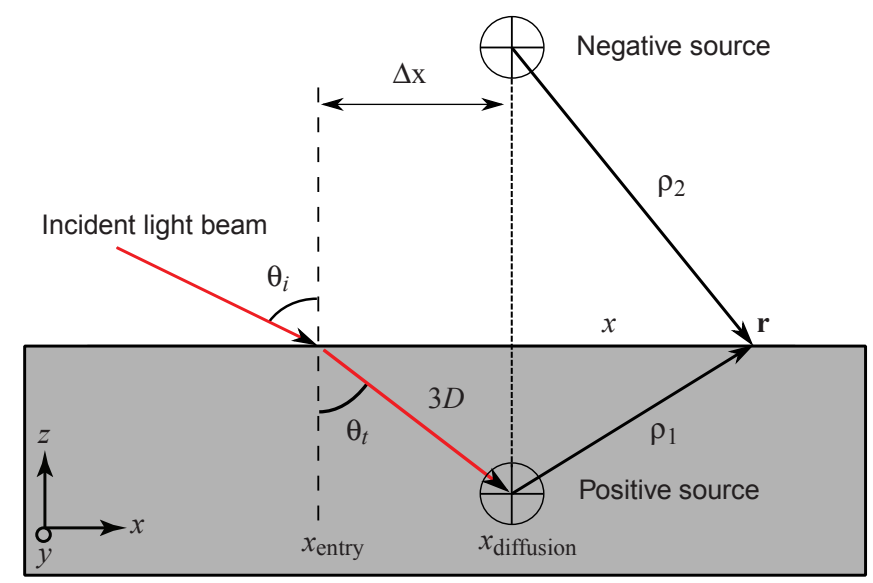

Fig. 2. Schematic presentation of OIR. The incoming light is refracted at the sample surface. The model describes the emergent intensity of light at the point $\mathbf{r}$ as a sum of contributions from a positive and a negative point source at distances $\rho_{1}$ and $\rho_{2}$. The two point sources are shifted horizontally by a distance $\Delta \mathrm{x}$ from the entry point of the light. 
where $\rho_{1}$ and $\rho_{2}$ are the distances from the positive source and the negative source to the location $\mathbf{r}$ on the sample surface, $\mu_{\text {eff }}$ is the effective transport coefficient. These sources are positioned at vertical distances of $z_{1}=3 D \cos \theta_{\mathrm{t}}$ and $z_{2}=z_{1}+$ $4 A D$. The parameter $A$ corrects the position of the negative source to account for internal reflection from the boundary back into the sample. It is approximated by ${ }^{12}$

$$
\begin{aligned}
\mathrm{A} & =\frac{1+F}{1-F} \\
F & =0.0636 n+0.668+0.710 n^{-1}-1.440 n^{-2} .
\end{aligned}
$$

To measure the optical properties, the diffusion coefficient $D$ is found first by estimating the distance between the entry point and the diffusion center (Eq. 1). Then $D$ is inserted in Eq. 3 and $\mu_{\text {eff }}$ is found by a nonlinear least squares fit of the equation to measured data. Combining this, the optical properties may be deduced ${ }^{15}$ :

$$
\begin{aligned}
\mu_{\mathrm{a}} & =D \mu_{\mathrm{eff}}^{2} \\
\mu_{\mathrm{s}}^{\prime} & =(3 D)^{-1}-0.35 \mu_{\mathrm{a}} .
\end{aligned}
$$

The technique thus imposes two criteria on the detection in relation to the optical properties of the sample

1. The reflectance profile must be resolved with sufficiently high resolution to expose the horizontal shift $\Delta \mathrm{x}$.

2. The field of view must be sufficiently wide such that the light becomes diffuse enough to match the diffusion expression in Eq. 3.

The range of optical properties that can be quantified robustly are therefore dependent on the detector system. One important aspect of the data collection is therefore that the reflectance profiles are completely resolved without saturated pixels. To accomplish this, together with a low noise measurement of the diffused light, the original implementation used two images of the reflectance. One images was taken with a neutral density filter to avoid saturation of the pixels close to the entry point. This had the undesirable side effect of shifting the position of the beam on the sample surface, which caused a systematic measurement error. Sun et al. ${ }^{21}$ used only one image with no filter applied to the source. This limits the range of the optical properties which can be measured, as highly scattering samples may saturate the CCD, and low scattering causes a low with noise.

With an image of the reflectance profile recorded, the calculation of the light's entry point has been handled in different ways. In the original OIR technique, ${ }^{14}$ the entry point is identified as the pixel of highest intensity. The pixel with highest intensity may however be a noisy estimate, especially in the presence of speckle which arises in narrow bandwidth systems. To improve the measurement precision, Sun et al. ${ }^{21}$ used a weighted center of mass of all pixels above the average intensity. Lindbergh et al. ${ }^{20}$ used the most intense pixel, but after a convolution with a Gaussian filter. To reduce noise even further, the average value of five repetitive measurements was used.

Similarly, different approaches have been taken for calculation of the diffusion center. It was originally estimated as the position at which the center line for the reflectance profile converged ${ }^{14}$ see illustration in Fig. 1b. For each point in the reflectance profile, the corresponding position on the other side is calculated by linear interpolation on a logarithmic scale of two points with the closets intensities above and below. The center of the two positions, on opposite sides of the entry point, becomes a point in the center line. In this way, the center line gives an estimation of the reflectance center at different intensity levels of the profile. The diffusion center is at the location where the center line converges. However, camerabased data requires a smoothing of this otherwise noisy profile. To improve the estimate of the diffusion center, Sun et al. ${ }^{21}$ used an iterative method. The location of the diffusion center was moved as well as the region of the image for which the diffusion fit was evaluated. Both were sequentially updated until a minimum change in the deduced optical properties was reached. The diffusion center was calculated as the symmetry point for the reflectance profiles of two intervals positioned symmetrically around the previous diffusion center. This iterative approach involves an additional nested loop for deducing the optical properties, and is thus slow. Lindbergh et al. ${ }^{20}$ computed a number of threshold-filtered images, where the thresholds were different percentages of the maximum intensity. The diffusion center was calculated as the evenly weighted center of mass of the pixels above the threshold. The intensity levels are only used if the light is sufficiently diffuse. This was quantified by the ratio of two second order moments for pixels above the threshold. Working on the entire images with iterative or multiple thresholds is time expensive, and scales with the camera resolution to the power of two.

The presented measurement procedure satisfies two important properties for an in-line inspection system. The measurement can be obtained through a cover slip, thus compatible with the hygiene requirements in dairy production. Another important aspect of the measurement technique is that the optical properties are measured in reflection mode, as opposed to transmission, which allows inspection of large volumes such as milk fermentation tanks. Compared with other diffuse reflectance spectroscopy instruments, ${ }^{15,20,24}$ the presented instrument has the broadest spectral and spatial range presented so far.

\section{METHOD}

This section describes the suggested solutions to the challenges of camera-based OIR (see the list of challenges in Table I).

Camera Intensity Range (C.1). To fully resolve the profiles, high dynamic range imaging is used. ${ }^{25}$ This means that several images are taken with different exposure times and these are combined into a single high dynamic range (HDR) image. The procedure described by Nielsen et al. ${ }^{26}$ is employed. Lindbergh et al. ${ }^{20}$ used a similar approach, but instead calibrated the response function to obtain HDR reflectance images. To ensure a linear response of the pixel value from the light intensity, the most intense pixels are interpreted as saturated. The actual value in these pixels is instead measured at a shorter exposure time using the HDR imaging. 
(a)
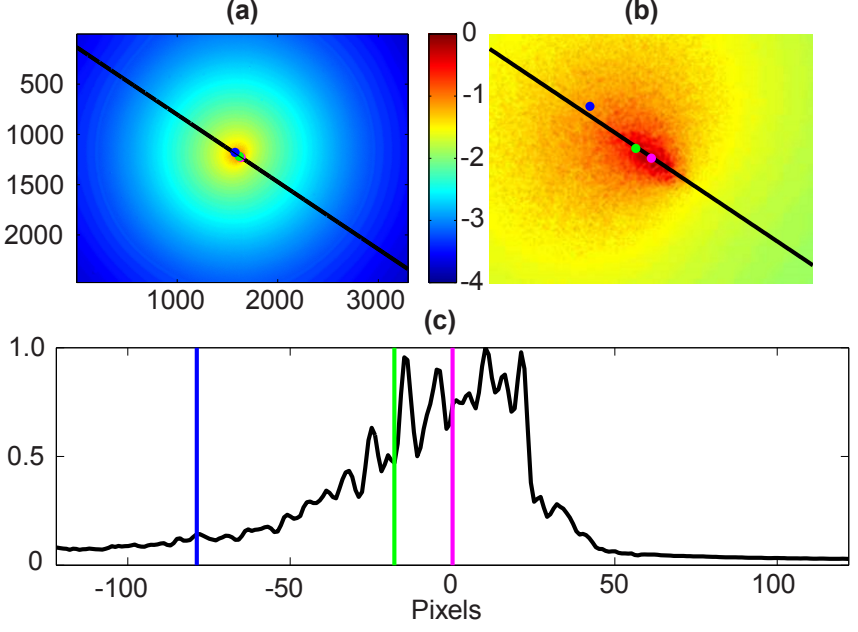

Fig. 3. The center estimation and line extracted from a HDR image of Arla Lærkevang $1.5 \%$ fat at $750 \mathrm{~nm}$. Intensity is normalized, and the color map uses a logarithmic scale. (a) HDR image, the blue marker is the center determined with the method by Sun et al., ${ }^{21}$ and the green shows the results of using the method by Lindbergh et al. ${ }^{20}$ The black line is the extracted line used for estimation of the entry point marked with magenta. (b) Zoom in on the beam's entry point in (a). (c) The extracted reflectance profile together with the projected entry points from $(\mathbf{b})$.

Entry Point and Laser Speckle (C.2 and C.3). To obtain an accurate estimate, a temporary center position is obtained as the median coordinate of pixels above $40 \%$ of the maximum image intensity. Subsequently, the reflectance profile along the direction of incidence is extracted for analysis. The angle of the horizontal axis in the image plane with the direction of incidence is measured during the assembly of the instrument, and the line is marked with black in Fig. 3. Reflectance values are extracted along this line, and the most intense pixel is the entry point. To reduce the noise of this estimate further, the average position over all wavelengths is used.

Estimating the Diffusion Center (C.4). As in the work of Lin et al., ${ }^{15}$ a single profile in the image is used rather than processing the entire image. However, due to the noise properties of the CCD, it is necessary to extend the work of Lin et al. so that it is possible to deal with non-monotonously decreasing data. To obtain a high accuracy estimate of the diffusion center, the overall development of the center line is captured using a regularized quadratic spline. Splines are advantageous as they do not enforce a specific shape of center lines as opposed to a closed expression. The regularization ensures robustness against noise in the image data. Finally, due to the quadratic nature, its derivatives are smooth, which is ideal for estimating the convergence of the profiles towards the location of the diffusion center. The diffusion center is calculated as the position of the spline at which its derivative as a function of intensity is zero. If the derivative is zero in more than one position, the one of the highest image intensity is chosen.

Examples of calculated center lines as a function of intensity depth are presented in Fig. 4a each together with its associated spline marked with black. Considering that the center
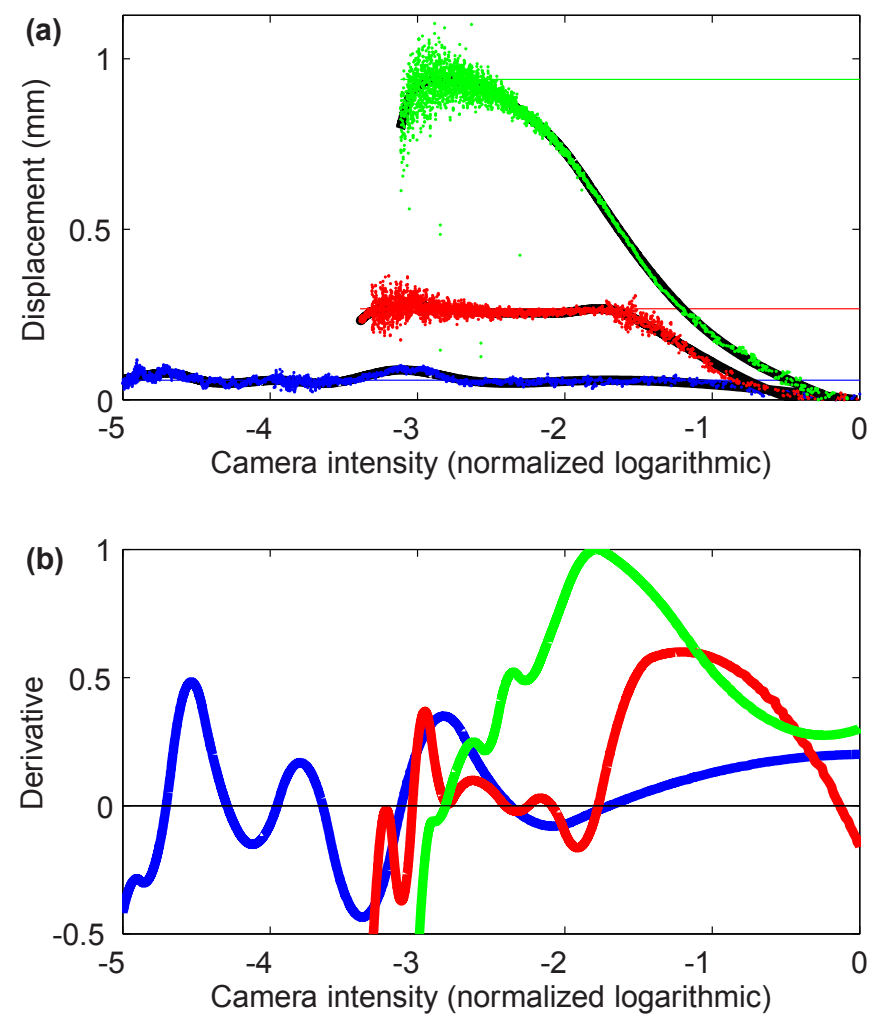

Fig. 4. Calculation of diffusion centers for Arla A38 3.5\% at $500 \mathrm{~nm}$ (blue), Arla Lærkevang 1.5\% at $970 \mathrm{~nm}$ (red) and Arla Lærkevang $0.5 \%$ at $970 \mathrm{~nm}$ (green). (a) The measured diffusion center lines presented as dots and a spline presented as the black line through each. The position of the diffusion center is marked as a vertical line. (b) The normalized derivative of the spline from the center lines, these are used to calculate the convergence of the lines

lines consist of roughly 3.5 thousand points, the development as a function of intensity is fairly well defined. The derivative of the splines used for estimating the convergence is seen in Fig. 4b. The diffusion center is evaluated as the point at which the derivative of the spline first crosses zero. At lower intensity values, the center line may be more subject to experimental factors such as sample roughness and unintended tilt between surface normal and camera axis.

Charge-Coupled Device (CCD) Pixel Calibration and Vignetting (C.5 and C.6). As OIR relies on intensity and shape, it is essential to characterize both lens distortion and vignetting. To quantify lens distortion, a calibration grid is placed on top of a homogeneous scattering sample. The light beam illuminates the sample similar to a measurement. The diffuse reflectance effectively provides a background illumination of the grid as seen in Fig. 5a. The image of the grid was enhanced by removing the smooth and diffuse part of the reflectance profile. This is done using a local noise normalization and dynamic range reduction of the image. From this the grid is annotated manually and the distance between points was fitted with a linear model. An example of the fit residual is presented in Fig. 5b. This procedure is carried out at 480 , 650 , and $970 \mathrm{~nm}$. From this, it was found that the image distortion is less than 2.5 pixels across the entire field of view, 
(a)
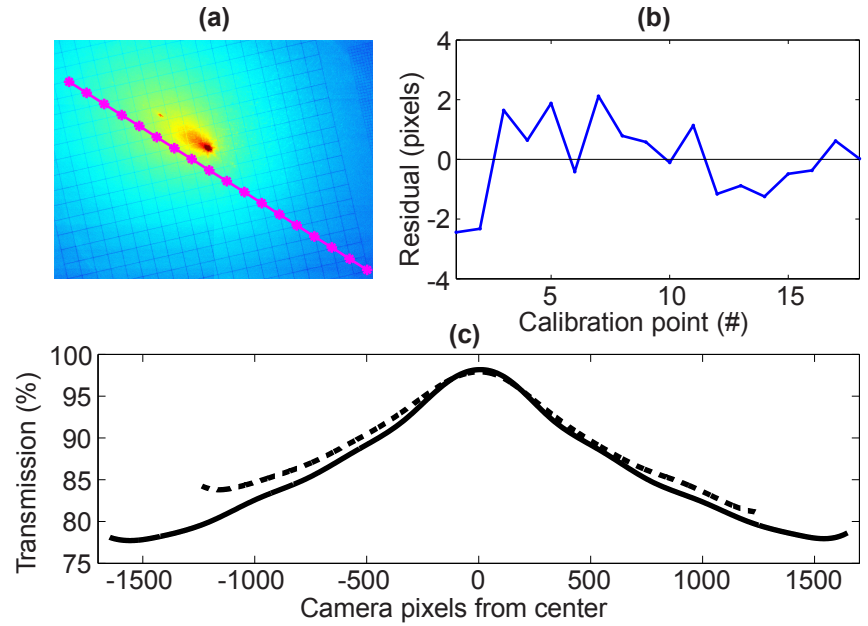

Fig. 5. (a) Image of the calibration grid on a logarithmic scale. (b) Residual in pixel distance from the model. (c) Vignetting along the two camera axes through the center.

which causes changes significantly below the measurement precision. Thus, no additional correction for lens distortion is required. The chromatic aberration, causing a wavelengthdependant image magnification, is quantified as less than $1 \%$. This approach is advantageous as it provides hyper spectral information and enables quantification of not only average pixel size but also possible image distortion.

To quantify the vignetting of the imaging system, an image is captured of a white paper poster smoothly illuminated by sunlight. The poster was positioned at a distance of around $1.5 \mathrm{~m}$ from the lens so that it was completely out of focus. This measurement shows a drop of transmission through the lens to about $80 \%$ toward the peripheral parts of the image. Traces of the vignetting signal are presented in Fig. 5c. This signal is used to correct all images captured to measure optical properties.

Fitting the Diffusion Model (C.7). The last unanswered question for the evaluation of the fit is the area excluded around the entry point and diffusion center. The reflected light in this area is interpreted as being insufficiently diffuse to be described by the diffusion equation. As noted by Lin et al. ${ }^{15}$ an advantage of OIR is that the area may be quantified in units of the transport mean free path mfp', which is calculated from $\Delta \mathrm{x}$ (see Fig. 2). Lin et al. ${ }^{15}$ report that the measured optical properties for low scattering samples are especially sensitive to this excluded area, and they used an area with a radius of 1.5 mfp'. Their work was based on fiber probes, which limited the possibility of a more general analysis. The presented method does not suffer from the same limitation due to the high spatial resolution of the presented camera-based instrumentation. The analysis is important in two regards: ${ }^{27}$

(I) The previously suggested value may not be optimal.

(II) How does the width of the excluded region affect the deduced optical properties?

Parts of the reflectance profile are excluded if they are too close to either the entry point or the diffusion center to be modeled as a diffusion process. This requirement is quantified by the following criterion:

$$
\max \left(\left|x-x_{\text {entry }}\right|,\left|x-x_{\text {diffusion }}\right|\right)<\left(\kappa \cdot \mathrm{mfp}^{\prime}+1.5 \cdot \omega_{\text {beam }}\right) .
$$

Here, $x$ is a point along the line extracted from the image data as seen in Fig. 3. The beam waist is denoted by $\omega_{\text {beam }}$, and $\kappa$ is the number of mfp' that are excluded. The factor $\kappa$ thus quantifies the width of the reflectance profile that is not described by the diffusion approximation.

One reason for these dependencies on the fit region is the analytical expression (Eq. 3) which is known to break down in two regimes: highly anisotropic scattering phase function ${ }^{28,29}$ and high absorption. ${ }^{30}$ Recently, corrections have been proposed to include phase function dependencies by Zemp. ${ }^{27}$ In addition, an improved analytical solution to the radiative transfer equation for a semi-infinite medium has recently been proposed by Liemert and Kienle. ${ }^{31}$ These two approaches may individually reduce the coupling between the optical properties. A combination of these approaches may provide a more general and adequate numerical model that describes the optical properties. However, in the present work, only the standard OIR model (Eq. 3) is considered.

Measurement of the Effective Attenuation. The second parameter used for determining the optical properties is the effective attenuation $\left(\mu_{\mathrm{eff}}\right)$, which is deduced from the shape of the diffused light by Eq. 3. Minimization of the following exression (Eq. 9) is used. Lin $^{32}$ used a similar expression but did not include beam convolution.

$$
\chi=\frac{1}{N} \sum_{n=1}^{N}\left(\frac{\int_{3 \cdot w_{\text {beam }}}^{-3 \cdot w_{\text {beam }}} \mathrm{R}\left(x_{n}-x\right) d x-y_{n}}{y_{n}}\right)^{2} .
$$

Here, $N$ denotes the number of points along the line of incidence, marked in black in Fig. 3, which fulfill the criterion in Eq. 8. The function $R$ denotes the diffuse reflectance intensity calculated by Eq. 3, and is evaluated in position $x_{n}$. The integral over $x$ is a convolution due to the finite beam size with waist $w_{\text {beam }}$. Lastly $y_{n}$ denotes intensity at a given pixel. Using this expression, each pixel only contributes with an error relative to the magnitude of the intensity. The two optimal values are determined by minimization using the LevenbergMarquardt algorithm.

Interpretation of Optical Properties. For the reduced scattering coefficients, the wavelength dependence is fitted with an approximation derived from Lorenz-Mie theory and presented by Graaff et al. ${ }^{33}$ This is done by fitting the model

$$
\mu_{s}^{\prime}=\mathrm{A} \lambda^{-\beta} \text {. }
$$

Here, $\lambda$ is the wavelength in $\mathrm{nm}, A$ and $\beta$ are fitted coefficients. The fitted amplitude coefficient $A$ indicates the concentration of the scatterers and their scattering cross sections, and the decay rate $\beta$ reflects the size parameter of the scatterers. In this way, wavelength resolved measurements of the reduced scattering coefficients enable discrimination between an increase in scattering particles density (A) from their size $(\beta)$.

For the wavelength dependence of the absorption coefficients, consult the spectrum of water reported by Hale and Querry ${ }^{34}$ and, for milk fat, the spectrum reported by Michalski et al. ${ }^{35}$ 


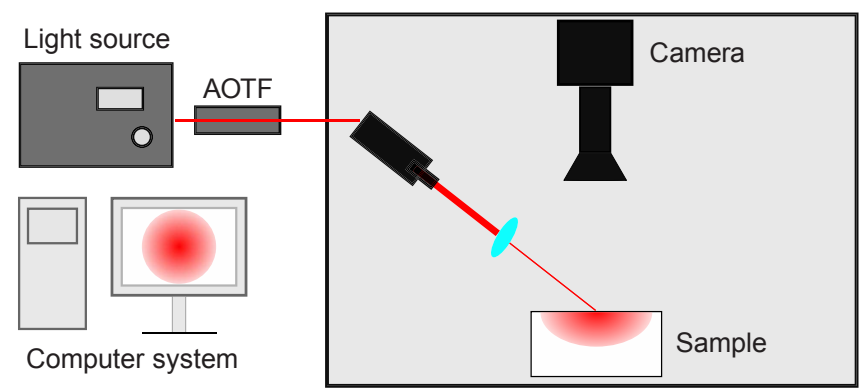

Fig. 6. Illustration of the diffuse reflectance instrument. The supercontinuum laser generates a broad spectral light profile and the specific wavelengths transmitted are selected using an acousto optical filter (AOTF). The beam, is focused onto the sample using an achromat. Images are captured of the light refletance profile by a CCD camera mounted above the sample.

\section{MATERIALS}

Hyperspectral Imaging System. Figure 6 illustrates the presented instrument for OIR measurements. The instrument is based on acousto-optical filtering of a broadband supercontinuum light source (SuperK Extreme EXW-12, NKT Photonics A/S, Birkerød, Denmark). The supercontinuum light source generates a beam throughout the spectral range from 450 to $2400 \mathrm{~nm}$ and for the present work, the acousto-optical filter (SuperK SELECT, NKT Photonics A/S, Birkerød, Denmark) was equipped with a crystal that enables wavelength selection in the spectral range from 475 to $1020 \mathrm{~nm}$. The optical bandwidth changes linearly from around $3.5 \mathrm{~nm}$ to roughly $16 \mathrm{~nm}$. To accomplish this broad spectral range, the filtering amplitude is lowered in the wavelength range above $900 \mathrm{~nm}$. The acousto-optically filtered light is coupled into a fiber delivery system (FDS; VIS-nIR FDS, NKT Photonics A/S, Birkerød, Denmark). The combined instrument produces a collimated single mode beam with a tunable wavelength which is ideal for OIR.

The FDS provides a collimated beam, and the beam size is reduced further using a lens. To keep the lens and its mounting equipment outside the camera field of view, the beam is focus on the sample surface using a $38 \mathrm{~mm}$ focal length achromatic lens (Edmund Optics Inc., Barrington, New Jersey, USA). The waist of the beam is wavelength-dependent and grows from approximately $120 \mu \mathrm{m}$ at $480 \mathrm{~nm}$ to $200 \mu \mathrm{m}$ at $1000 \mathrm{~nm}$; this includes the effect of the angular projection. The incidence angle of the beam is $\theta_{i}=52^{\circ}$, which was found as a compromise between increasing the diffusion displacement (Eq. 1) while keeping the projected beam small and having transmission into the sample. More detailed considerations for incidence angle and polarization direction are provided by Abildgaard. ${ }^{36}$

Images of the reflectance profiles are captured using a 12bit CCD camera (AM-800GE, JAI, Miyazaki, Japan) with a pixel resolution of $3296 \times 2472$. The imaging objective is a $50 \mathrm{~mm}$ focal length lens (LM50XC, Kowa Company Ltd., Nagoya, Japan), mounted to provide an image with a resolution of $3.2 \mu \mathrm{m}$ per pixel of the sample surface. The shutter on
TABLE II. Product information for the dairy products. The size parameters are typical values and measured by a Mastersizer 3000 (Malvern, United Kingdom) evaluated with a refractive index of 1.529 , the instrument does not support multiple constituents as is the case for milk.

\begin{tabular}{lcccc}
\hline & \multicolumn{2}{c}{ Fat } & \multicolumn{2}{c}{ Protein } \\
& $\begin{array}{c}\text { Content } \\
(\mathrm{g} / 100 \mathrm{~g})\end{array}$ & $\begin{array}{c}\text { Mode } \\
(\mu \mathrm{m})\end{array}$ & $\begin{array}{c}\text { Content } \\
(\mathrm{g} / 100 \mathrm{~g})\end{array}$ & $\begin{array}{c}\text { Mode } \\
(\mu \mathrm{m})\end{array}$ \\
\hline Product name & 3.5 & 0.99 & 3.4 & 0.046 \\
Arla Lærkevang Sødmælk & 1.5 & 0.99 & 3.5 & 0.046 \\
Arla Lærkevang Letmælk & 0.5 & 0.99 & 3.5 & 0.052 \\
Arla Lærkevang Minimælk & 0.5 & & 3.4 & \\
\hline Arla A38 naturel 3.5\% & 3.5 & & 3.8 & \\
Arla A38 naturel 1.5\% & 1.5 & & 4.3 & \\
Arla A38 naturel 0.5\% & 0.5 & & & \\
\hline
\end{tabular}

the objective was set to $\mathrm{F} 7$ and magnification to 2 . To calibrate the imaging system, a calibration grid (R1L3S3p, Thorlabs, Gothenburg, Sweden) was used.

Dairy Samples. The measurement technique was evaluated using a series of dairy products. This series contains commercially available milk and yogurt with three different fat levels. The names of the products and key information on their composition are in Table II. Mean particle size values are not available for fermented milks as the necessary sample dilution destroys the gel network. ${ }^{37}$ All products were purchased during December 2013 in Copenhagen, Denmark. The yogurt products are fermented with the bacteria culture $L$. acidophilus. The milk and yogurt products have very different structural properties because the casein protein in yogurts contain a gel network rather than being suspended individual micelles. The chemical composition in terms of fat and protein contents is largely unchanged, especially for the $3.5 \%$ products where the difference is only in the microstructure of the protein gel.

By inspecting the samples presented in Table II the instrument sensitivity is evaluated as the discrimination between the deduced optical properties of similar milk and yogurt products. The measurement precision of all dairy products was quantified as the standard deviation between five repetitive measurements. The measurement series was performed with 11 spectral bands.

Measurement reproducibility. It is commonly recognised that there is a strong correlation between the optical properties. ${ }^{30,38}$ To investigate the correlation between the deduced scattering and absorption coefficient, the optical properties of Arla Lærkevang 1.5\% fat was measured 19 times and deduced using a value of $\kappa=2$.

\section{RESULTS}

Hyperspectral Imaging System (C.1 and C.4). An example HDR image is presented in Fig. 3a, which shows a smoothly changing profile across regions acquired with different exposure times. Since the optical properties are de- 


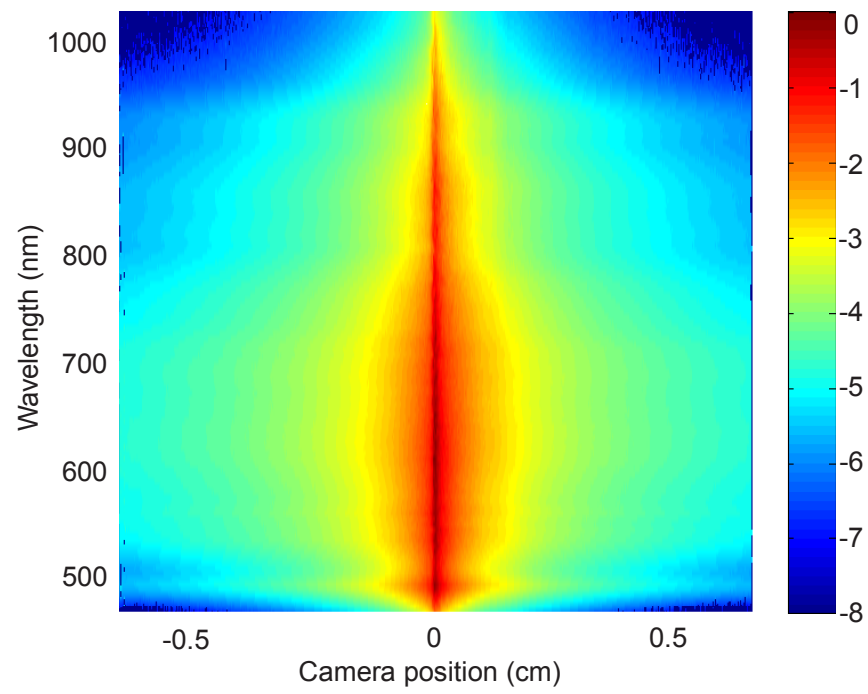

Fig. 7. Combined spectrograph image of the reflectance profiles for Arla $\mathrm{A} 38^{\circledR}$ naturel $3.5 \%$. Color map shows the reflectance intensity on 10 base logarithmic scale.

duced from a line through the image, the entire hyperspectral information used to estimate the optical properties may be illustrated as a spectrograph image, see Fig. 7. The image data is normalized to the most intense pixel over the entire spectrum. Spectrograph images may be used to assess the dynamics of the instrument, where it is seen that the signal is preserved throughout the entire spectrum. It also shows that the dynamic range of intensities for this dairy product spans $80 \mathrm{~dB}$. The broad dynamic range can not be covered by any CCD chip and the measurements are only possible due to the HDR procedure.

The data acquisition time for an HDR image is $1.9 \mathrm{~s}$ per wavelength but may be reduced, as the same shutter times are used for all wavelengths and samples. From the spectrograph images (Fig. 7 is an example), it is evident that some of the long exposure shutter times for the wavelength range $550-$ $800 \mathrm{~nm}$ could be skipped as the signal for these is stronger. Similarly, the short shutter times may be omitted for spectra in the blue and NIR range.

Finally, the spectrograph image (Fig. 7) may be used to identify absorption bands from the raw data directly. Absorption gives contraction of the reflectance profile over a short wavelength range. In Fig. 7, an absorption band is present from around $950 \mathrm{~nm}$ and upwards. Some absorption is also observable around $520 \mathrm{~nm}$. In comparison, the scattering causes a slow monotonous decay.

Estimating the Entry Point (C.2 and C.3). For the present instrument and samples, the speckle structure seen in Fig. 3 surrounds the entry point. Entry points obtained with techniques proposed in previous work ${ }^{15,21}$ are presented in Fig. 3 together with the entry point obtained with the method presented here. The speckle pattern shifts the location of the most intense pixel away from the entry point, not only along the direction of incidence. For the present measurement, the location of the most intense pixel shifts by approximately 15 pixels. Previous methods for reducing the effects of speckle overshoot in the present data, see Fig. 3c. The reason is that these methods rely on estimation of the centers of symmetric distributions. This causes an underestimation of $\Delta x$ and leads to an overestimation of the scattering coefficient and underestimation of the absorption coefficient. As opposed to previous work, the presented method only searches for the most intense pixel along the line of incidence and further reduces variance by averaging across wavelength. In this way, an unbiased estimate of the entry point is obtained.

Estimating the Diffusion Center (C.4). The fact that the diffusion center is determined from a single line of reflectance values increases the range of optical properties that may be estimated. For samples with little scattering and absorption, the asymmetric area of the reflectance is larger and two-dimensional methods rely on convergence of the asymmetry within the image. Such methods are thus limited by the field of view along the shortest side of the image. This limitation was acknowledged by Lindbergh et al. ${ }^{20}$ who excluded samples due to lack of dynamic range. The 1D method further extends the range, because the measurements may be conducted with the line of incidence along the diagonal of the image, which increases the number of pixels along the line. In addition, the computation speed is significantly faster and scales better with the camera resolution.

Fit and Foot Print of the Diffusion Model (C.5, C.6, and C.7). Previous work reports that the deduced optical properties depend on the range of the fit interval. ${ }^{15,21}$ To investigate the significance of this effect on the dairy samples, the deduced optical properties and the fit residual are quantified with varying width ( $\kappa)$ of the excluded data during the fit to Eq. 9. The value of $\kappa$ is varied from 0.5 to 5 and the effect on both the deduced optical properties and fit residual is investigated. An example is presented in Fig. 8, where it is seen that the deduced absorption coefficient depends on the region where the data is fitted. The deduced absorption changes smoothly, and reaches maximum at $\kappa=2.2$. The reduced scattering on the other hand is largely unchanged. It is noteworthy that the minimum fit error occurs at $\kappa=1.4$, which is different from where the absorption peaks. These effects have been investigated across wavelength and sample. In general, it was found that the reduced scattering coefficient is indeed independent of $\kappa$. The deduced absorption coefficient does, however, depend on $\kappa$, but does not in general have a maximum.

It is of interest to investigate whether an optimal $\kappa$ value exists. The visual agreement between fit and selected data from the presented results does not, however, give clear indication of which value to choose. The example in Fig. 8 also indicates this. All values of $\kappa$ seem to give a decent description of the data. The results show that the dependence on $\kappa$ is not trivial. As seen from the example in Fig. 8, the deduced absorption and fit residual do not converge at large values of $\kappa$. Neither does a single value of $\kappa$ exist at which the fit residual is minimised and absorption maximised. This could otherwise have been a criterion for finding an optimal value of $\kappa$.

The fit residual for the camera calibration presented in Fig. 5b clearly shows that the image distortion is not causing systematic dependence of the optical properties on the fit region presented in Fig. 8. Nevertheless, the presented high precision method for spectral characterization of the lens dis- 
(a)

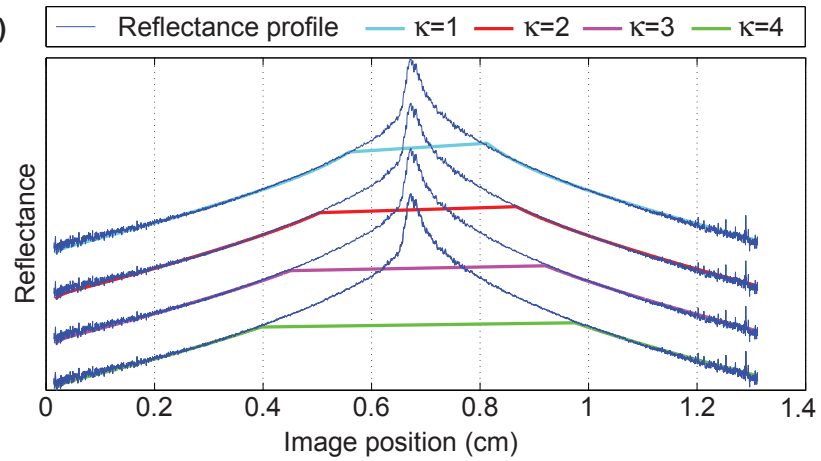

(b)

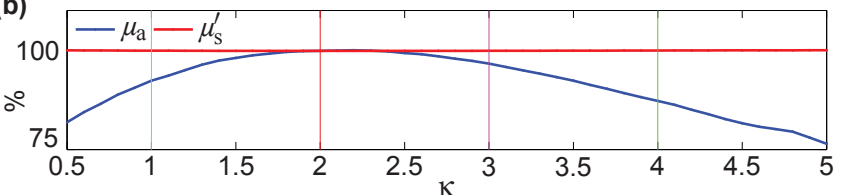

(c)

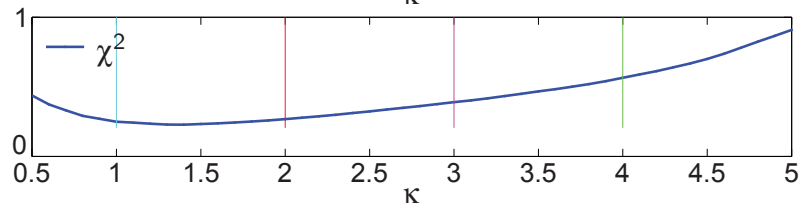

Fig. 8. Example of how the foot print affects the fit for Arla A38 naturel $0.5 \%$ at $970 \mathrm{~nm}$. (a) Examples of the fitted profiles in colour. (b) Deduced absorption coefficient (red) and reduced scattering (blue). (c) Fit residuals.

tortion is a necessary validation. Concerning the image vignetting, this effect is normalized after the images are captured. If this normalization is undesirable, vignetting may be reduced by replacing the existing lens with a more expensive telecentric lens.

A final remark on the dependence on fit range $(\kappa)$ is that the specific value mainly influences the absorption coefficient which is important when the measurements are used for chemometric evaluation of the sample constituent. For the purpose of quantifying the reduced scattering coefficient, and hence the particle size distribution, which changes during milk fermentation, the specific value of $\kappa$ is not essential.

Spectra for Dairy Samples. To investigate the performance of the combined instrument and analysis technique in characterizing the optical properties of dairy products, the optical properties of the samples in Table II were measured (using $\kappa=2$ ). The resulting curve fits for some of the most diverse reflectance profiles of the dairy products are presented in Fig. 9, together with their respective fits. This gives an impression of how well the data is modeled with the proposed measurement technique. Measurement of all products was repeated 5 times, and the mean and standard deviation of the deduced optical properties are summarized in Fig. 10.

The fit results presented in Fig. 9 demonstrate the fit performance for the investigated dairy products. These results show a fairly good characterization of the reflectance profiles across both the diverse milk and yogurt samples and the wavelength range from blue to NIR. However, it is evident that the amount of data to fit the diffusion model with milk $0.5 \%$ is somewhat limited.

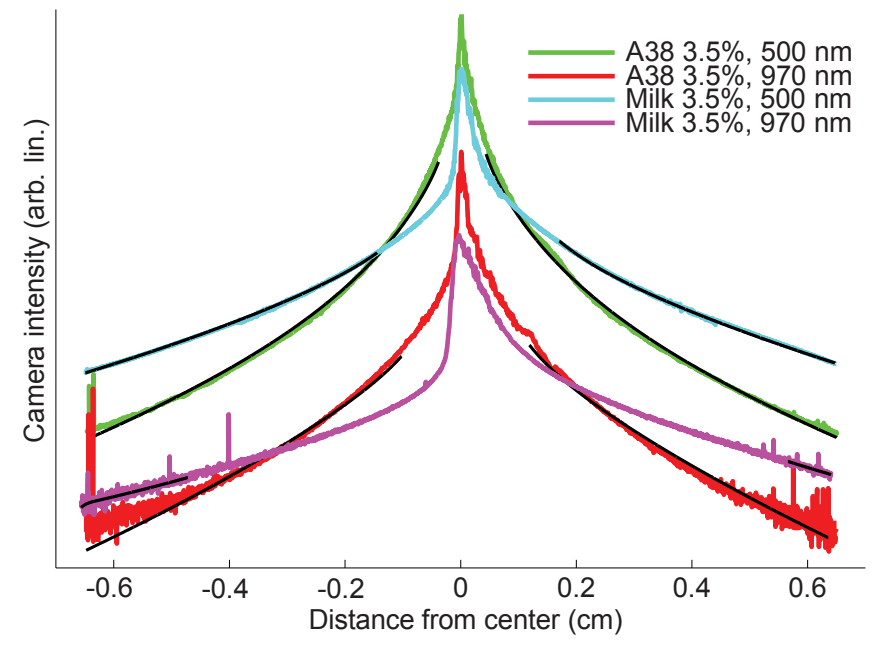

Fig. 9. Examples of reflectance profiles and their fitted effective attenuation (black lines).

The measured reduced scattering coefficient, shown in Fig. 10, increases as a function of fat content. This is as expected since fat particles are the main cause for scattering. Similar results have been reported for other measurement techniques. ${ }^{5,24}$

Concerning the discrimination of the structural difference between milk and fermented milk products, the evaluated optical properties of the dairy samples are compared directly in Fig. 10. From this, it is seen that the reduced scattering properties provide a clear distinction between the two products. Furthermore, it is seen that the values are in agreement with the approximate development derived from Lorenz-Mie theory and previously reported by Nielsen et al. ${ }^{5}$ The measurement precision of the fermented products is in general lower than the milk products. This is due to their deflation and slightly curved surface.

In the measured absorption spectra, three peaks are present throughout all the measurements. The first peak is around $520 \mathrm{~nm}$ and has previously been reported for milk fat, ${ }^{35}$ the presence of a weak peak at $750 \mathrm{~nm}$ and a stronger peak at $970 \mathrm{~nm}$ have previously been observed for water. ${ }^{34}$ In general, the results show a reduction of the measured absorption for the fermented products. This effect may be a result of the scattering phase function of the samples. This induces a cross-talk between the absorption and scattering properties. Lastly, with respect to the range of optical properties that may be covered, it is seen that the limited field of view for the present system reduces the absorption estimate for the Arla Lærkevang ${ }^{\circledR}$ Minimælk $0.5 \%$ fat.

Measurement Reproducibility. A scatter plot of the deduced optical properties is presented in Fig. 11 for a single wavelength. The correlation coefficient is -0.94 , which indicates high correlation of the noise contribution in scattering and absorption. It is probable that the systematic variations are largely induced through the estimation of the diffusion displacement. This error then propagates on through the curve fitting procedure, where the estimated effective attenuation compensates for the error in mean free path estimation. 
Reduced scattering $(1 / \mathrm{cm})$

Fat: $3.5 \%$

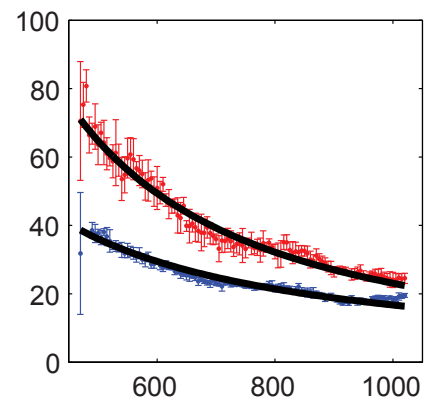

Fat: $1.5 \%$

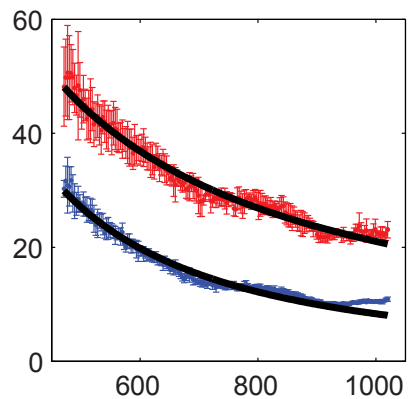

Fat: $0.5 \%$

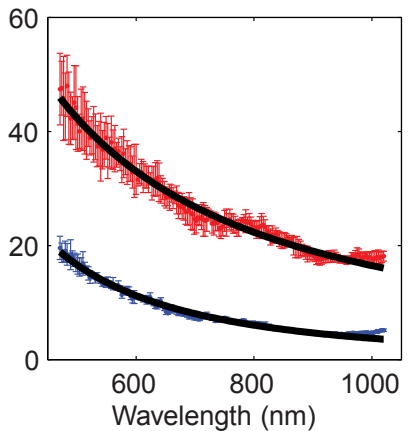

Absorption (1/cm)
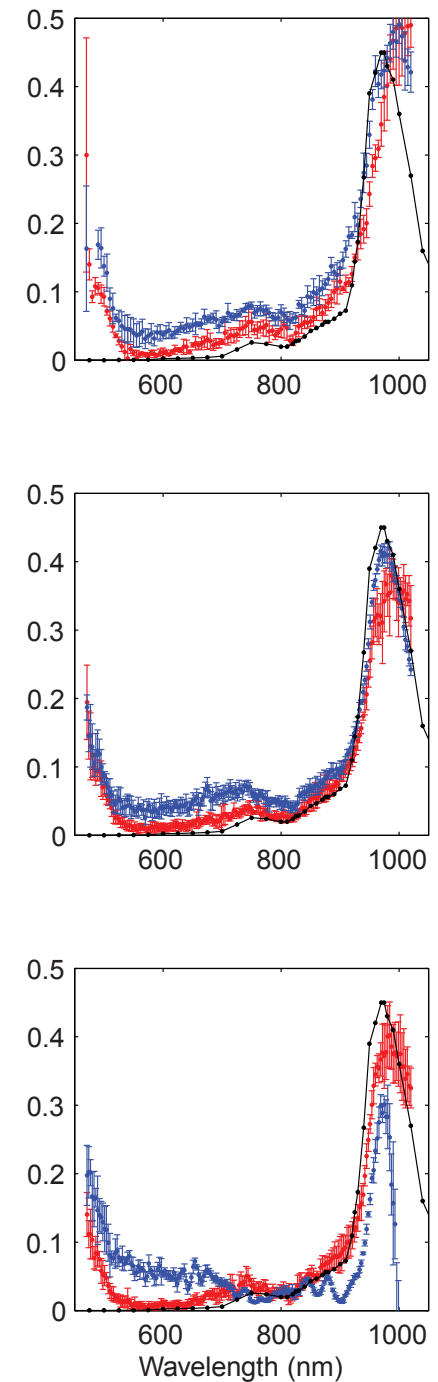

Fig. 10. Measured optical properties for dairy products with 5 repetitive measurements showing the standard deviation at each wavelength. Red data is for fermented milk products, blue is for milk. For the reduced scattering coefficients, the black lines are fits to Equation 10. For the absorption measurements, the black line is the absorption of water. ${ }^{34}$

These results suggest that the presented method has potential to become a method with even higher precision in estimation of optical properties if the error in estimation of the transport mean free path can be reduced further.

\section{CONCLUSION}

The development of new optical characterization techniques is of great interest for the dairy industry. In the present work, a camera-based instrument for quantifying the optical properties of milk and fermented milk has been demonstrated. Measurements are performed by only quantifying light reflection from the sample surface. This makes it useful for in-

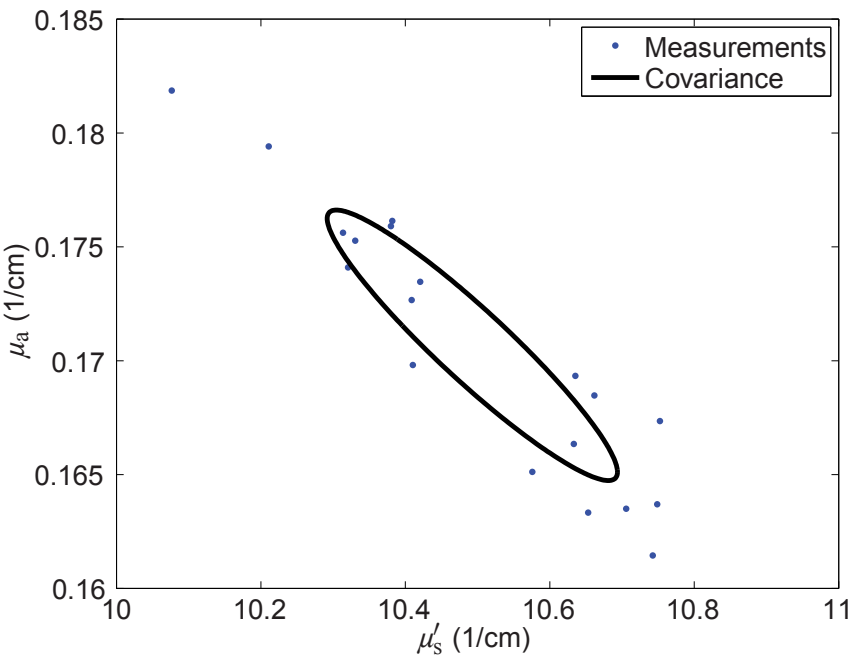

Fig. 11. Scatterplot of the estimated optical properties of 19 repetitive measurements of milk $1.5 \%$ fat at $930 \mathrm{~nm}$, the ellipsoid shows the circle span by the eigenvalue decomposition of the data's covariance.

line measurements, despite large sample volume, as in a milk fermentation tank. In addition, the technique enables a noncontact and non-invasive measurement, which is important for avoiding contamination and accomplishing an automated measurement system.

The instrument covers the broad spectral range from 470 to $1020 \mathrm{~nm}$ and thus covers the absorption bands of both milk fat and water together with the reduced scattering properties of the samples. The optical properties of commercially available milk and fermented milk has been reported and show strong capabilities of discriminating between initial and final state of fermented milk products by their reduced scattering coefficient. Simultaneously, the absorption coefficient clearly marks the presence of both fat and water but also a correlation to the scattering levels.

\section{Acknowledgements}

This work was financed by the center for Imaging Food Quality project which is funded by the Danish Council for Strategic Research (contract no 09-067039) within the Program Commission on Health, Food and Welfare.

1. F. G. C. Bijnen, H. van Aalst, P. Baillif, J. C. G. Blonk, D. Kersten, F. Kleinherenbrink, R. Lenke, and M. M. vander Stappen, "In-line structure measurement of food products," Powder Technology 124, 188-194 (2002).

2. W. Labs, "Inline monitoring aids in food safety and quality: What you don't measure could kill your brand," Food Engineering Magazine 85, 51-52, 54, 56-59 (2013).

3. T. Janh $\varnothing$ j, C. B. Petersen, M. B. Frøst, and R. Ipsen, "Sensory and rheological characterization of low-fat stirred yogurt," Journal of Texture Studies 37, 276-299 (2006).

4. M. Auty, M. Fenelon, T. Guinee, C. Mullins, and D. Mulvihill, "Dynamic confocal scanning laser microscopy methods for 
studying milk protein gelation and cheese melting," Scanning 21, 299-304 (1999).

5. O. H. A. Nielsen, A. A. Subash, F. D. Nielsen, A. B. Dahl, J. L. Skytte, S. Andersson-Engels, and D. Khoptyar, "Spectral characterisation of dairy products using photon time-of-flight spectroscopy," Journal of Near Infrared Spectroscopy 21, 375-383 (2013).

6. B. M. Agrawal and M. Mengüç, "Forward and inverse analysis of single and multiple scattering of collimated radiation in an axisymmetric system," International Journal of Heat and Mass Transfer 34, 633 - 647 (1991).

7. M. Alexander and D. Dalgleish, "Dynamic light scattering techniques and their applications in food science," Food Biophysics 1, 2-13 (2006).

8. S. Sharifzadeh, J. Skytte, L. Clemmensen, and B. Ersbøll, "DCTbased characterization of milk products using diffuse reflectance images," in "The 18th International Conference on Digital Signal Processing (DSP)," (2013), pp. 1-6.

9. J. L. Skytte, O. H. A. Nielsen, U. Andersen, J. M. Carstensen, A. L. Dahl, R. Larsen, F. Møller, F. Kamran, and J. R. Frisvad, "Decomposition of diffuse reflectance images - features for monitoring structure in turbid media," Proceedings of the InsideFood Symposium 2013 (2013).

10. M. Navrátil, C. Cimander, and C.-F. Mandenius, "On-line multisensor monitoring of yogurt and filmjölk fermentations on production scale," Journal of Agricultural and Food Chemistry 52, 415-420 (2004).

11. C. Soukoulis, P. Panagiotidis, R. Koureli, and C. Tzia, "Industrial yogurt manufacture: Monitoring of fermentation process and improvement of final product quality," Journal of Dairy Science 90, 2641-2654 (2007).

12. T. J. Farrell, M. S. Patterson, and B. Wilson, "A diffusion theory model of spatially resolved, steady-state diffuse reflectance for the noninvasive determination of tissue optical properties in vivo," Medical Physics 19, 879-888 (1992).

13. Y. Peng and R. Lu, "An LCTF-based multispectral imaging system for estimation of apple fruit firmness. part 2. selection of optimal wavelengths and development of prediction models." Transactions of the ASAE 49, 269-275 (2006).

14. L. Wang and S. L. Jacques, "Use of a laser beam with an oblique angle of incidence to measure the reduced scattering coefficient of a turbid medium," Applied Optics 34, 2362-2366 (1995).

15. S.-P. Lin, L. Wang, S. L. Jacques, and F. K. Tittel, "Measurement of tissue optical properties by the use of oblique-incidence optical fiber reflectometry," Applied Optics 36, 136-143 (1997).

16. A. Garcia-Uribe, J. Zou, M. Duvic, J. H. Cho-Vega, V. G. Prieto, and L. V. Wang, "In vivo diagnosis of melanoma and nonmelanoma skin cancer using oblique incidence diffuse reflectance spectrometry," Cancer Research 72, 2738-2745 (2012).

17. A. Garcia-Uribe, K. Balareddy, J. Zou, and L. Wang, "Micromachined fiber optical sensor for in vivo measurement of optical properties of human skin," IEEE Sensors Journal 8, 1698-1703 (2008).

18. A. Garcia-Uribe, E. B. Smith, J. Zou, M. Duvic, V. Prieto, and L. V. Wang, "In-vivo characterization of optical properties of pigmented skin lesions including melanoma using oblique incidence diffuse reflectance spectrometry," Journal of Biomedical Optics 16, 020501-020501-3 (2011).

19. G. Marquez and L. Wang, "White light oblique incidence reflectometer for measuring absorption and reduced scattering spectra of tissue-like turbid media," Optics Express 1, 454 (1997).

20. T. Lindbergh, M. Larsson, I. Fredriksson, and T. Strömberg, "Reduced scattering coefficient determination by non-contact oblique angle illumination: methodological considerations," Proceedings of SPIE, Optical Interactions with Tissue and Cells XVIII 6435, 64350I-64350I-12 (2007).

21. P. Sun, X. Cao, R. Yang, F. Xie, J. Ding, and F. Zhang, "Basic research on determining optical properties of tissues in vivo by measuring diffuse reflectance with a charge-coupled device," Optica Applicata 3, 541-555 (2011).

22. A. J. Jääskeläinen, K.-E. Peiponen, and J. A. Räty, "On reflectometric measurement of a refractive index of milk," Journal of Dairy Science 84, 38-43 (2001).

23. J. Ripoll, D. Yessayan, G. Zacharakis, and V. Ntziachristos, "Experimental determination of photon propagation in highly absorbing and scattering media," Journal of the Optical Society of America A 22, 546-551 (2005).

24. J. Qin and R. Lu, "Measurement of the absorption and scattering properties of turbid liquid foods using hyperspectral imaging," Applied Spectroscopy 61, 388-396 (2007).

25. P. E. Debevec and J. Malik, "Recovering high dynamic range radiance maps from photographs," in "Proceedings of SIGGRAPH 97,', (1997), pp. 369-378.

26. O. H. A. Nielsen, A. L. Dahl, R. Larsen, F. Møller, F. D. Nielsen, C. L. Thomsen, H. Aanæs, and J. M. Carstensen, "In depth analysis of food structures, hyperspectral subsurface laser scattering," in "Proceesings of Scandinavian Workshop on Imaging Food Quality, Ystad, Sweden,” (2011), pp. 27-32.

27. R. J. Zemp, "Phase-function corrected diffusion model for diffuse reflectance of a pencil beam obliquely incident on a semi-infinite turbid medium," Journal of Biomedical Optics 18, 067005-067005 (2013).

28. L. V. Wang and S. L. Jacques, "Source of error in calculation of optical diffuse reflectance from turbid media using diffusion theory," Computer Methods and Programs in Biomedicine 61, $163-170$ (2000).

29. A. Kienle, F. K. Forster, and R. Hibst, "Influence of the phase function on determination of the optical properties of biological tissue by spatially resolved reflectance," Optics Letters 26, 1571-1573 (2001).

30. F. Martelli, S. Del Bianco, A. Ismaelli, and G. Zaccanti, Light propagation through biological tissue and other diffusive media: theory, solutions, and software (SPIE Press Bellingham, 2010).

31. A. Liemert and A. Kienle, "Exact and efficient solution of the radiative transport equation for the semi-infinite medium," Scientific Reports 3, 1-7 (2013).

32. S.-P. Lin, "Oblique-incidence fiber-optic reflectometry for measuring absorption and scattering in turbid media," Master's thesis, Rice University (1996).

33. R. Graaff, J. G. Aarnoudse, J. R. Zijp, P. M. A. Sloot, F. F. M. de Mul, J. Greve, and M. H. Koelink, "Reduced light-scattering properties for mixtures of spherical particles: a simple approximation derived from mie calculations," Applied Optics 31, 1370-1376 (1992).

34. G. M. Hale and M. R. Querry, "Optical constants of water in the 200-nm to 200- $\mu \mathrm{m}$ wavelength region," Applied Optics 12, 555-563 (1973).

35. M.-C. Michalski, V. Briard, and F. Michel, "Optical parameters of milk fat globules for laser light scattering measurements," Le Lait 81, 787-796 (2001).

36. O. H. A. Abildgaard, "Broadband optical characterization of material properties," Ph.D. thesis, Technical University of Denmark (2014).

37. W. J. Lee and J. A. Lucey, "Formation and physical properties of yogurt," Asian-Aust. J. Anim. Sci. 23, 1127-1136 (2010).

38. L. V. Wang and H.-I. Wu, Biomedical Optics: Principles and Imaging (Wiley, 2007). 